\title{
Bose-Einstein condensation in a two-dimensional, trapped, interacting gas
}

\author{
M. Bayindir and B. Tanatar \\ Department of Physics, Bilkent University, Bilkent, 06533 Ankara, Turkey
}

(Received 1 April 1998)

\begin{abstract}
We study the Bose-Einstein condensation phenomenon in a two-dimensional (2D) system of bosons subjected to a harmonic-oscillator-type confining potential. The interaction among the $2 \mathrm{D}$ bosons is described by a $\delta$ function in configuration space. Solving the Gross-Pitaevskii equation within the two-fluid model we calculate the condensate fraction, ground-state energy, and specific heat of the system. Our results indicate that interacting bosons have similar behavior to those of an ideal system for weak interactions.
\end{abstract}

[S1050-2947(98)00210-8]

PACS number(s): 03.75.Fi, 05.30.Jp, 67.40.Kh

The observation of the Bose-Einstein condensation (BEC) phenomenon in dilute atomic gases [1-4] has caused a lot of attention, because it provides opportunities to study the thermodynamics of weakly interacting systems in a controlled way. The condensate clouds obtained in the experiments consist of a finite number of atoms (ranging from several thousands to several millions), and are confined in externally applied confining potentials. The ground-state properties of the condensed gases, including the finite size effects on the temperature dependence of the condensate fraction, are of primary interest. At zero temperature, the mean-field approximation provided by the Gross-Pitaevskii equation [5] describes the condensate rather well and at finite temperatures a self-consistent Hartree-Fock-Bogoliubov (HFB) approximation is developed [6]. Path integral Monte Carlo (PIMC) simulations [7] on three-dimensional, interacting bosons appropriate to the current experimental conditions demonstrate the effectiveness of the mean-field-type approaches. Various aspects of the mean-field theory, as well as detailed calculations corresponding to the available experimental conditions, are discussed by Giorgini et al. [8].

In this work we examine the possibility of BEC in a twodimensional (2D) interacting atomic gas, under a trap potential. Such a system may be realized by making one dimension of the trap very narrow so that the oscillator states are largely separated. Possible experimental configurations in spin polarized hydrogen and magnetic waveguides are currently under discussion [9]. The study of 2D systems is also interesting theoretically, since even though the homogeneous system of $2 \mathrm{D}$ bosons does not undergo BEC [10], a number of examples [11] have indicated such a possibility upon the inclusion of confining potentials. We employ the two-fluid, mean-field model developed by Minguzzi et al. [12] to study the 2D Bose gas. Similar approaches [13] are gaining attention because of their simple and intuitive content; these approaches also provide semianalytical expressions for the density distribution of the condensate. Recently, Mullin [14] considered the self-consistent mean-field theory of 2D Bose particles interacting via a contact interaction within the Popov and semiclassical approximations. His conclusions were that a phase transition occurs for a 2D Bose system, in the thermodynamic limit, at some critical temperature, but not necessarily to a Bose-Einstein condensed state. However, in the current experiments the finite number of atoms $N$ prevents various divergences from giving rise to behavior akin to noninteracting systems.

Our work is motivated by the success of mean-field, twofluid models $[12,13]$ vis à vis more involved calculations and direct comparison with experiments. In the following we briefly describe the two-fluid model of Minguzzi et al. [12] and present our results for the 2D Bose gas.

The condensate wave function $\Psi(r)$ is described by the Gross-Pitaevskii (GP) equation [5]

$$
\begin{aligned}
- & \frac{\hbar^{2}}{2 m} \nabla^{2} \Psi(r)+V_{\mathrm{ext}}(r) \Psi(r) \\
& +2 g n_{1}(r) \Psi(r)+g \Psi^{3}(r)=\mu \Psi(r),
\end{aligned}
$$

where $g$ is the repulsive, short-range interaction strength, $V_{\text {ext }}(r)=m \omega^{2} r^{2} / 2$ is the confining (or trap) potential, and $n_{1}(r)$ is the distribution function for the noncondensed particles. We note that unlike in a three-dimensional system, $g$ in our case is not simply related to the $s$-wave scattering length, but will be treated as a parameter. In the two-fluid model developed by Minguzzi et al. [12] the noncondensed particles are treated as bosons in an effective potential $V_{\text {eff }}(r)=V_{\text {ext }}(r)+2 g n_{1}(r)+2 g \Psi^{2}(r)$, and having the same chemical potential $\mu$ with that of the condensate. The density distribution is given by

$$
n_{1}(r)=\int \frac{d^{2} p}{(2 \pi \hbar)^{2}} \frac{1}{\exp \left\{\left[p^{2} / 2 m+V_{\text {eff }}(r)-\mu\right] / k_{B} T\right\}-1},
$$

and the chemical potential is fixed by the relation

$$
N=N_{0}+\int \frac{\rho(E) d E}{\exp \left[(E-\mu) / k_{B} T\right]-1},
$$

where $N_{0}=\int \Psi^{2}(r) d^{2} r$ is the number of condensed atoms, and the semiclassical density of states is calculated using $[12,15,16]$

$$
\rho(E)=\frac{m}{2 \pi \hbar^{2}} \int_{V_{\mathrm{eff}}(r)<E} d^{2} r .
$$




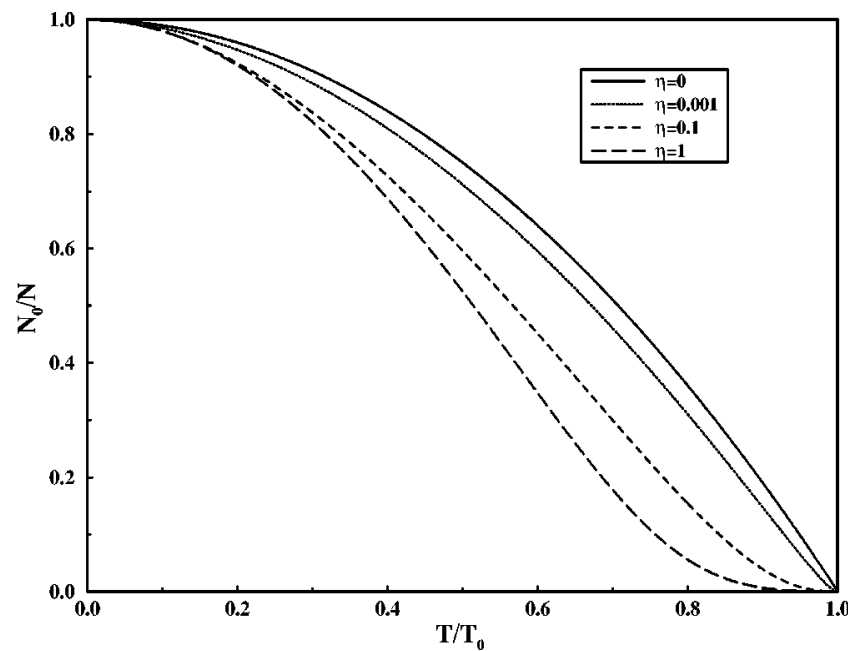

FIG. 1. Condensate fraction $N_{0} / N$ as a function of $T / T_{0}$, for a system of $N=10^{5}$ atoms. The various interaction strengths are described by the parameter $\eta$.

The GP equation admits a simple solution within the Thomas-Fermi approximation, i.e., when the kinetic energy term is neglected,

$\Psi^{2}(r)=\frac{1}{g}\left[\mu-V_{\mathrm{ext}}(r)-2 g n_{1}(r)\right] \theta\left(\mu-V_{\mathrm{ext}}(r)-2 g n_{1}(r)\right)$,

where $\theta(x)$ is the unit step function. Thomas-Fermi approximation is regarded to be rather good except for the region close to the phase transition [17]. Minguzzi et al. [12] have numerically solved the above set of equations selfconsistently. They have also introduced a simpler approximation scheme which treats the interaction effects perturbatively. Encouraged by the success of even the zero-order solution in describing the fully numerical self-consistent solution in the 3D case, we attempt to look at the situation in 2D. In a similar vein, we treat the interactions among the noncondensed particles perturbatively. To zero order in $g n_{1}(r)$, the number of condensed particles is calculated to be

$$
N_{0}=\frac{\pi \hbar^{2}}{g m}\left(\frac{\mu}{\hbar \omega}\right)^{2}
$$

and the density of states is obtained as

$$
\rho_{0}(E)= \begin{cases}E /(\hbar \omega)^{2} & \text { if } \mu<0 \\ 2(E-\mu) /(\hbar \omega)^{2} & \text { if } 2 \mu>E \quad(\mu>0) \\ E /(\hbar \omega)^{2} & \text { if } 2 \mu<E \quad(\mu>0) .\end{cases}
$$

If we use the above form of the density of states, valid for $E>0$, then we obtain

$$
N=N_{0}+t^{2}\left[\frac{\pi^{2}}{3}-\operatorname{dilog}\left(1-e^{-\alpha / t}\right)\right],
$$

where $t=k_{B} T / \hbar \omega$, and $\alpha=\mu / \hbar \omega$. The chemical potential $\mu(N, T)$ is obtained as the solution of this transcendental equation.

In Fig. 1 we show the temperature dependence of the

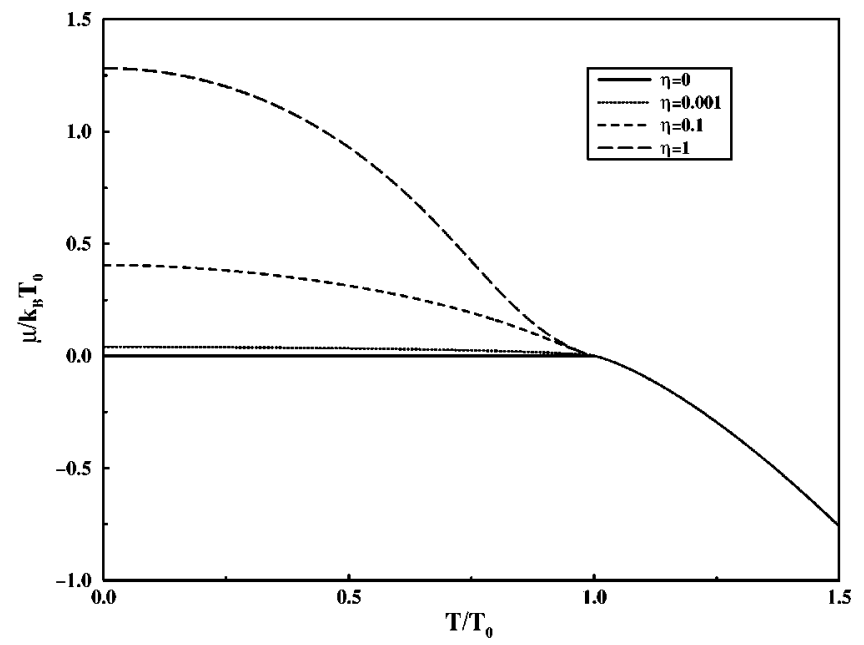

FIG. 2. Temperature dependence of the chemical potential $\mu$ for various interaction strengths.

condensate fraction $N_{0} / N$ for a system of $N=10^{5}$ particles, and for various values of the interaction strength. Also shown for comparison is the result for an ideal 2D Bose gas in a harmonic trap, given by $N_{0} / N=1-\left(T / T_{0}\right)^{2}$ where $k_{B} T_{0}=\hbar \omega[N / \zeta(2)]^{1 / 2}$. We observe that BEC-like behavior occurs for small values of the parameter $\eta=m g / \pi \hbar^{2}$, i.e., the weakly interacting system. Here we identify the BEC with the macroscopic occupation of the ground state at $T$ $=0$ and the depletion of it above $T_{0}$. As the strength of interactions is increased we find that the temperature dependence of $N_{0} / N$ deviates from the noninteracting case more noticeably. Mullin [14] has argued that there is no BEC in $2 \mathrm{D}$ in the thermodynamic limit. We consider a system with finite number of particles, and we were able to obtain a selfconsistent solution for the chemical potential for various values of the interaction strength as displayed in Fig. 2. We next evaluate the temperature dependence of the internal energy $\langle E\rangle=\left[\langle E\rangle_{\mathrm{nc}}\left(N-N_{0}\right) / 2+\langle E\rangle_{c}\right] / N$ which consists of contributions from the noncondensed particles

$$
\begin{aligned}
\langle E\rangle_{\mathrm{nc}}= & k_{B} T_{0}\left(\frac{\zeta(2)}{N}\right)^{1 / 2} \\
& \times\left[\frac{\pi^{2} \alpha t^{2}}{3}+t^{3}\left(2 \zeta(3)+\int_{0}^{\alpha / t} \frac{x^{2} d x}{e^{x}-1}\right)\right. \\
& \left.-\alpha^{2} t \ln \left(1-e^{-\alpha / t}\right)\right],
\end{aligned}
$$

and the condensed particles

$$
\langle E\rangle_{c}=k_{B} T_{0} \frac{1}{3 \eta}\left(\frac{\zeta(2)}{N}\right)^{1 / 2} \alpha^{3} .
$$

In the above expressions $\zeta(n)$ is the Riemann zeta function. The kinetic energy of the condensed particles is neglected in accordance with our Thomas-Fermi approximation to the GP equation. In Fig. 3 we display the temperature dependence of $\langle E\rangle$ for different values of the interaction strength. The noninteracting energy is simply $\langle E\rangle / N k_{B} T_{0}=[\zeta(3) /$ $\zeta(2)]\left(T / T_{0}\right)^{5}$. For small $\eta$, and $T<T_{0}$, the behavior of $\langle E\rangle$ resembles that in a $3 \mathrm{D}$ system. As $\eta$ increases, a bump in 


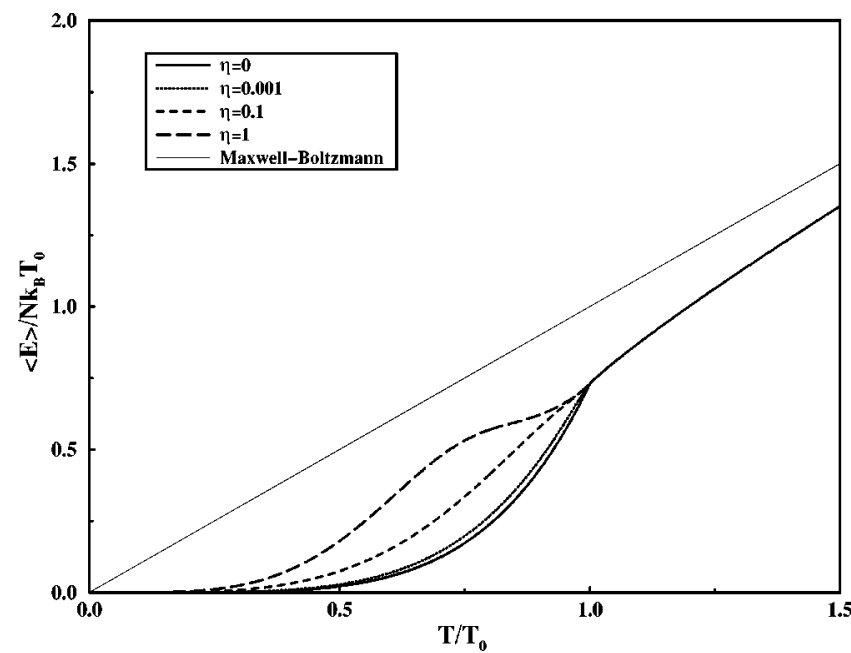

FIG. 3. Ground-state energy $\langle E\rangle$ of the 2D bosons as a function of temperature for various interaction strengths. The MaxwellBoltzmann result is shown by the thin solid line.

$\langle E\rangle$ develops for $T<T_{0}$, which perhaps indicates the breakdown of the present approximation or an artifact of the calculation. We have no physical explanation for this behavior. The corresponding results for the specific heat $C_{V}$ $=d\langle E\rangle / d T$ are shown in Fig. 4. In contrast to the noninteracting case where a sharp peak at $T=T_{0}$ is seen, the effects of short-range interactions smoothes out the transition. However, this smoothing is partly due to the finite number of particles in the system [8]. The effects of interactions and finite number of particles are not disentangled in our treatment.

It is a straightforward generalization to include the effects of anisotropy within the present formalism. For an external potential of the type $V_{\text {ext }}(r)=m \omega_{x}^{2}\left(x^{2}+\lambda^{2} y^{2}\right) / 2$, where $\lambda$ $=\omega_{y} / \omega_{x}$ is the anisotropy parameter, both $N_{0}$ and $\rho_{0}(E)$ depend inversely on $\lambda$. Similarly, our analysis may be extended to study other power law potentials such as $V \sim r^{\gamma}$ for which $\gamma \approx 1$ appears to be interesting $[18,19]$.

Our calculations using the two-fluid model of Minguzzi et al. [12] show that the BEC, in the sense of macroscopic occupation of the ground state, may occur in a 2D trapped Bose gas when the short-range interparticle interactions are not too strong. As the interaction strength increases we could

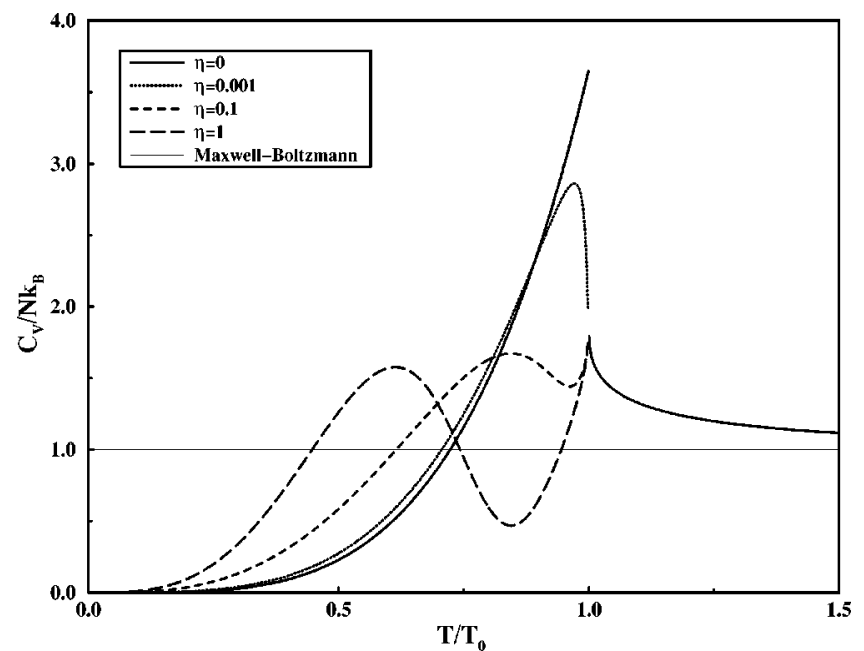

FIG. 4. Specific heat $C_{V}=d\langle E\rangle / d T$ as a function of temperature for various interaction strengths.

not find self-consistent solutions to the mean-field equations signaling the breakdown of our approach. We note that instead of using the lowest-order perturbation approach adopted here, the full solution to the self-consistent equations may alleviate the situation. Given the unclear nature of the phase transition $[14,18]$ in $2 \mathrm{D}$ and the interest of future experiments, we think it is worthwhile to perform firstprinciples calculations. Recent PIMC simulations [19] on 2D hard-core bosons confirm the possibility of BEC in the sense that a sharp drop in $N_{0} / N$ around $k_{B} T_{c} \approx 0.78 N^{1 / 2}$ is observed for finite systems.

In summary, we have applied the mean-field, semiclassical two-fluid model for trapped interacting Bose gases to the case in two dimensions. We have found that for a range of interaction strength parameters the behavior of the thermodynamic quantities resembles that of noninteracting bosons in a harmonic trap.

This work was partially supported by the Scientific and Technical Research Council of Turkey (TUBITAK). We thank Dr. S. Conti for providing us with the details of his calculations and Dr. S. Pearson for sending us a preprint of their work prior to publication. We gratefully acknowledge useful discussions with E. Keçecioğlu, H. Mehrez, and T. Senger.
[1] M. H. Anderson, J. R. Ensher, M. R. Matthews, C. E. Wieman, and E. A. Cornell, Science 269, 198 (1995).

[2] K. B. Davis, M.-O. Mewes, M. R. Andrews, N. J. van Druten, D. S. Durfee, D. M. Kurn, and W. Ketterle, Phys. Rev. Lett. 75, 3969 (1995); M.-O. Mewes, M. R. Andrews, N. J. van Druten, D. M. Kurn, D. S. Durfee, and W. Ketterle, ibid. 77, 416 (1996).

[3] J. R. Ensher, D. S. Jin, M. R. Matthews, C. E. Wieman, and E. A. Cornell, Phys. Rev. Lett. 77, 4984 (1996).

[4] C. C. Bradley, C. A. Sackett, and R. G. Hulet, Phys. Rev. Lett. 78, 985 (1997).

[5] E. P. Gross, Nuovo Cimento 20, 454 (1961); L. P. Pitaevskii,
Zh. Eksp. Teor. Fiz. 40, 646 (1961) [Sov. Phys. JETP 13, 451 (1961)].

[6] A. Griffin, Phys. Rev. B 53, 9341 (1996).

[7] W. Krauth, Phys. Rev. Lett. 77, 3695 (1996).

[8] S. Giorgini, L. P. Pitaevskii, and S. Stringari, Phys. Rev. A 54, 4633 (1996); J. Low Temp. Phys. 109, 309 (1997).

[9] I. F. Silvera, in Bose-Einstein Condensation, edited by A. Griffin, D. W. Snoke, and S. Stringari (Cambridge University Press, Cambridge, England, 1995); E. A. Hinds, M. G. Boshier, and I. G. Hughes, Phys. Rev. Lett. 80, 645 (1998).

[10] P. C. Hohenberg, Phys. Rev. 158, 383 (1967).

[11] A. Widom, Phys. Rev. 176, 254 (1968); R. Masut and W. J. 
Mullin, Am. J. Phys. 47, 493 (1979); V. Bagnato and D. Kleppner, Phys. Rev. A 44, 7439 (1991).

[12] A. Minguzzi, S. Conti, and M. P. Tosi, J. Phys.: Condens. Matter 9, L33 (1997).

[13] R. J. Dodd, K. Burnett, M. Edwards, and C. W. Clark, Acta Phys. Pol. A 93, 45 (1998); M. Naraschewski and D. M. Stamper-Kurn Phys. Rev. A (to be published)..

[14] W. J. Mullin, J. Low Temp. Phys. 110, 167 (1998).
[15] V. Bagnato, D. E. Pritchard, and D. Kleppner, Phys. Rev. A 35, 4354 (1987).

[16] G.-L. Ingold and A. Lambrecht, Eur. Phys. J. D 1, 29 (1998).

[17] G. Baym and C. J. Pethick, Phys. Rev. Lett. 76, 6 (1996).

[18] S. I. Shevchenko, Zh. Éksp. Teor. Fiz. 100, 1824 (1991) [Sov. Phys. JETP 73, 1009 (1991)].

[19] S. Pearson, T. Pang, and C. Chen (unpublished). 